\title{
A Bijection for Directed-Convex Polyominoes
}

\author{
Alberto Del Lungo, ${ }^{1}$ Massimo Mirolli, ${ }^{1}$ Renzo Pinzani, ${ }^{2}$ and Simone \\ Rinaldi $^{2}$ \\ ${ }^{1}$ Università di Siena, Dipartimento di Matematica, via del Capitano, 15, 53100, Siena, Italy [dellungo, \\ mirolli@unisi.it]. \\ ${ }^{2}$ Università di Firenze, Dipartimento di Sistemi e Informatica, via Lombroso, 6/17, 50134, Firenze, Italy [pinzani, \\ rinaldi@dsi.unifi.it].
}

received January 30, 2001, revised May 4, 2001, accepted May 16, 2001.

In this paper we consider two classes of lattice paths on the plane which use north, east, south, and west unitary steps, beginning and ending at $(0,0)$. We enumerate them according to the number of steps by means of bijective arguments; in particular, we apply the cycle lemma. Then, using these results, we provide a bijective proof for the number of directed-convex polyominoes having a fixed number of rows and columns.

Keywords: cycle lemma, directed-convex polyominoes, binomial coefficients, lattice paths.

\section{Introduction}

In the plane $Z \times Z$ the following four types of steps are taken into consideration: north steps, $(0,1)$, east steps, $(1,0)$, south steps, $(0,-1)$, and west steps, $(-1,0)$. Let $\mathcal{C}$ denote the set of all lattice paths which use north, east, south, and west steps, beginning and ending at $(0,0)$ (see Fig. 1 on page 2). Each path belonging to $\mathcal{C}$ has an even number of steps; for $n \geq 0$, let $\mathcal{C}_{2 n}$ denote the set of paths in $\mathcal{C}$ having $2 n$ steps. In this paper we will give a bijective proof that the cardinality of $C_{2 n}$ equals, for $n \geq 0$,

$$
\left(\begin{array}{c}
2 n \\
n
\end{array}\right)^{2}
$$

Let $\mathcal{C}^{+}\left(C_{2 n}^{+}\right.$, resp.) denote the subset of $\mathcal{C}\left(\mathcal{C}_{2 n}\right.$, resp.) whose paths remain weakly above the $x$-axis (see Fig. 2 on page 2). The path set $\mathcal{C}^{+}$was originally studied in [2], where the authors proved, for $n \geq 0$,

$$
\left|C_{2 n}^{+}\right|=\left(\begin{array}{c}
2 n \\
n
\end{array}\right)^{2}-\left(\begin{array}{c}
2 n \\
n+1
\end{array}\right)^{2}
$$

This result has been considered further by Guy, Krattenthaler, and Sagan in [8] and by Sulanke in [13]. 1365-8050 @ 2001 Maison de l'Informatique et des Mathématiques Discrètes (MIMD), Paris, France 


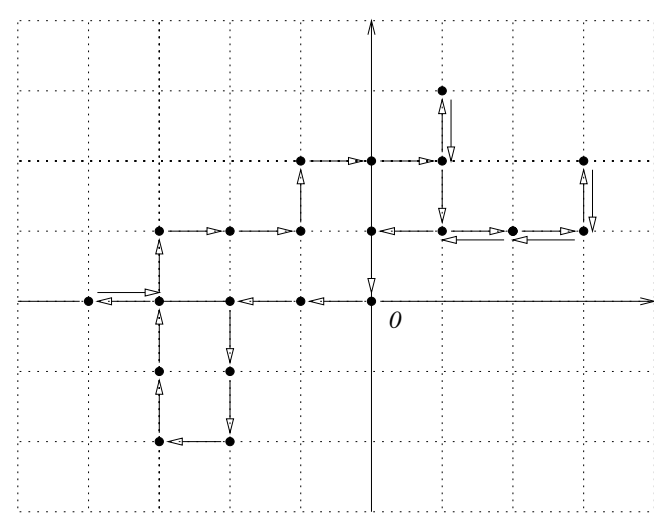

north step

east step

south step

west step

Fig. 1: A $C$ path with 26 steps.

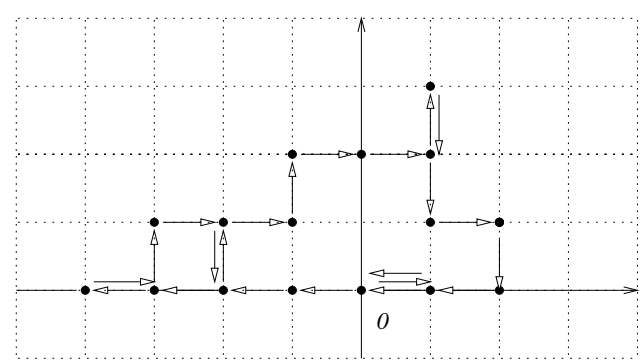

Fig. 2: A $C^{+}$path with 22 steps.

We prove this statement bijectively by applying the well-known cycle lemma, originally introduced in [6], and then rediscovered and applied many times as in [5] and [10]. In particular our proof first shows

$$
\left|\mathcal{C}_{2 n}^{+}\right|=\frac{2 n+1}{(n+1)^{2}}\left|\mathcal{C}_{2 n}\right|=\frac{1}{2 n+1}\left(\begin{array}{c}
2 n+1 \\
n
\end{array}\right)^{2}, \quad n \geq 0
$$

It is then straightforward to show that the formulas of (2) and (3) agree.

In the last part of the paper we consider the class of directed-convex polyominoes and the class of parallelogram polyominoes, each having $n+1$ columns and $n+1$ rows. Narayana [9] was the first to show, in essence, that the number of parallelogram polyominoes having $n+1$ columns and $n+1$ rows is equal to the number in (2). Chang and Lin [3], and later Bousquet-Mélou [1, p.111], proved that the number of directed-convex polyominoes having $n+1$ columns and $n+1$ rows is equal to the number in (1). In this paper we give a combinatorial proof of the previous statements by establishing bijections defined on the classes $\mathcal{C}^{+}$and $C$. 


\section{About cycles of 2-colored Motzkin paths}

The 2-colored Grand Motzkin paths are lattice paths that begin and end on the $x$-axis and use the rise step, $(1,1)$, the fall step, $(1,-1)$, and of two types of horizontal steps, $(1,0)$, namely the $\alpha$-colored and $\beta$-colored horizontal steps. It is easy to show that the cardinality of the set of 2-colored Grand Motzkin paths running from $(0,0)$ to $(n, 0)$ is the central binomial coefficient, $\left(\begin{array}{c}2 n \\ n\end{array}\right)$. The 2-colored Motzkin paths are Grand Motzkin paths that remain weakly above the $x$-axis. The number of 2-colored Motzkin paths of length $n$ is well known to equal the $(n+1)$ th Catalan number, [12, p.219].
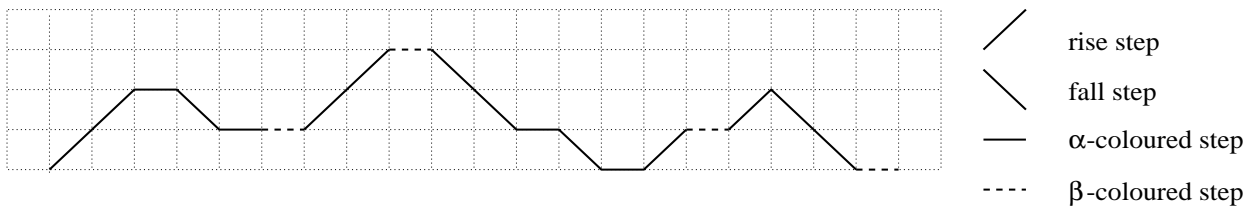

Fig. 3: A 2-colored Motzkin path with 20 steps.

We will call a 2-colored Grand Motzkin path having the same number of $\alpha$ and $\beta$ steps, a cycle. This name is suggested by the simple bijection between $\mathcal{C}_{2 n}$ and the set of Grand Motzkin paths having length $2 n$ that is achieved by the following coding:
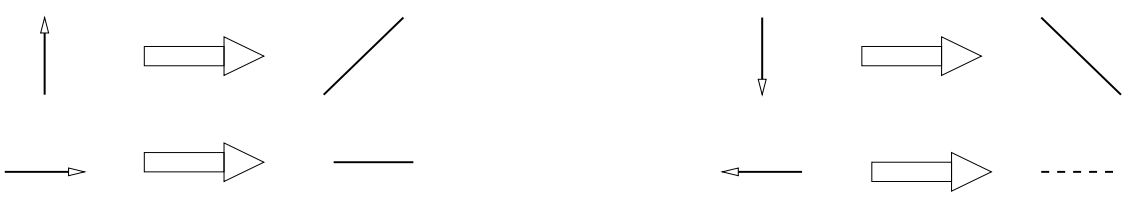

Fig. 4: The step transformation of paths of $\mathcal{C}_{2 n}$ into cycles of length $2 n$.

For example, the cycle represented in Figure 5 corresponds to the path of Fig. 1 on page 2.

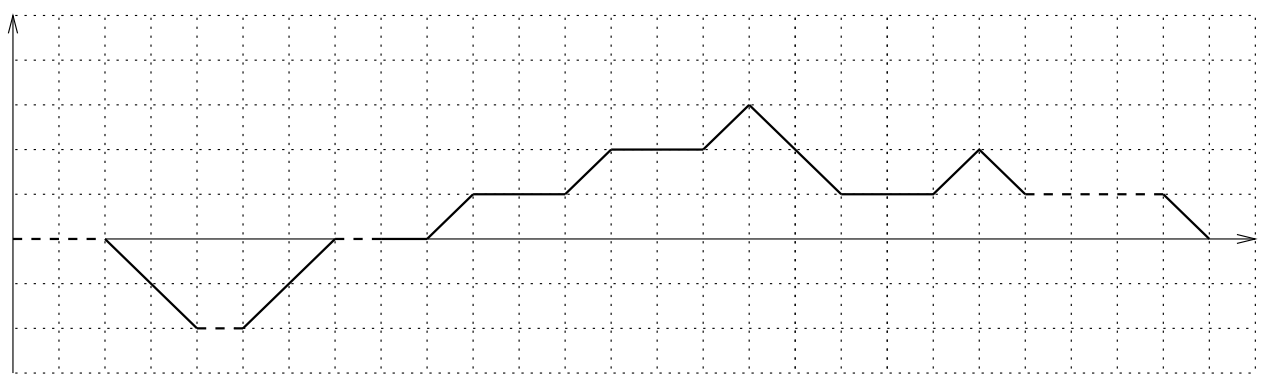

Fig. 5: A cycle having length 26. 
Lemma 1 The number of $2 n$-length cycles is equal to the central binomial coefficients squared,

$$
\left(\begin{array}{c}
2 n \\
n
\end{array}\right)^{2}
$$

Proof. To prove our claim, we will establish a correspondence between the cycles of length $2 n$ and Grand Dyck paths of length $4 n$ decomposable as pairs of Grand Dyck paths of length $2 n$. Let us consider a cycle of length $2 n$. We code each step of this cycle with a vector $2 \times 1$ :

$$
\begin{aligned}
& \left(\begin{array}{l}
1 \\
0
\end{array}\right) \text { for a rise step, } \quad\left(\begin{array}{l}
0 \\
1
\end{array}\right) \text { for a fall step, } \\
& \left(\begin{array}{l}
1 \\
1
\end{array}\right) \quad \text { for an } \alpha \text {-horizontal step, }\left(\begin{array}{l}
0 \\
0
\end{array}\right) \quad \text { for a } \beta \text {-horizontal step. }
\end{aligned}
$$

Therefore, we can represent the cycle by a $2 \times n$ matrix simply by concatenating the $n$ vectors corresponding to its steps. For example, the cycle of Fig. 5 on page 3 can be represented by the matrix:

$$
\left(\begin{array}{llllllllllllllllllllllllll}
0 & 0 & 0 & 0 & 0 & 1 & 1 & 0 & 1 & 1 & 1 & 1 & 1 & 1 & 1 & 1 & 0 & 0 & 1 & 1 & 1 & 0 & 0 & 0 & 0 & 0 \\
0 & 0 & 1 & 1 & 0 & 0 & 0 & 0 & 1 & 0 & 1 & 1 & 0 & 1 & 1 & 0 & 1 & 1 & 1 & 1 & 0 & 1 & 0 & 0 & 0 & 1
\end{array}\right) .
$$

Moreover, if we code a rise step by 1 and a fall step by 0 , then each row of the matrix is a Grand Dyck path. The concatenation of these two paths gives a Grand Dyck path of length $4 n$. The previously defined transformation can be simply inverted.

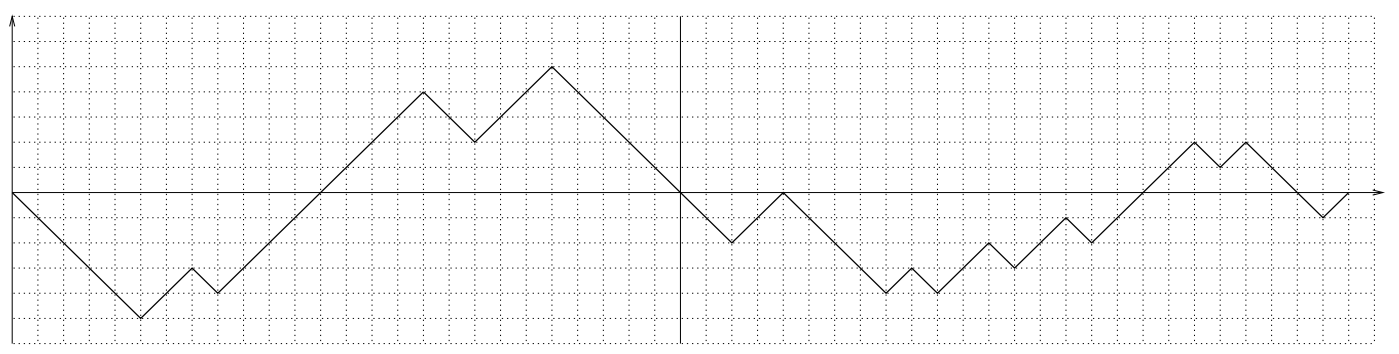

Fig. 6: The Grand Dyck path corresponding to the cycle of Fig. 5 on page 3.

Let us now examine the set of positive cycles, that is, the set of cycles that remain weakly above the $x$-axis. The coding of Fig. 4 ensures us that each path of $\mathcal{C}_{2 n}^{+}$corresponds to a positive cycle of length $2 n$. For example the path in Fig. 2 on page 2 corresponds to that in Fig. 7.

We now combinatorially prove that the number of positive cycles with $2 n$ steps is equal to

$$
\left|\mathcal{C}_{2 n}^{+}\right|=\frac{2 n+1}{(n+1)^{2}}\left|\mathcal{C}_{2 n}\right|=\frac{1}{2 n+1}\left(\begin{array}{c}
2 n+1 \\
n
\end{array}\right)^{2}, \quad n \geq 0 .
$$

(We leave the simple analytical proof of (5) to the reader.) Let $X_{2 n+1}, n \geq 0$ denote the class of paths using the same steps as the 2 -colored Motzkin paths, having the same number of $\alpha$-colored and $\beta$-colored steps, 


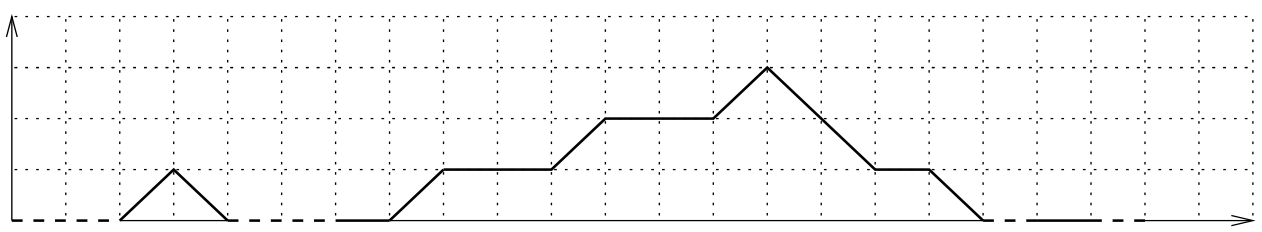

Fig. 7: The positive cycle corresponding to the path in Fig. 2 on page 2.

and running from $(0,0)$ to $(2 n+1,1)$. For any path in this class, the number of rise steps exceeds the number of fall steps by one unit. The same arguments used to prove Lemma 1 will convince the reader that

$$
\left|X_{2 n+1}\right|=\left(\begin{array}{c}
2 n+1 \\
n
\end{array}\right)^{2}
$$

To have the desired proof of (5) it is sufficient to show

$$
\left|X_{2 n+1}\right|=(2 n+1)\left|\mathcal{C}_{2 n}^{+}\right| \text {. }
$$

The proof of (7) will be neat application of the cycle lemma, as recorded in [7]:

Lemma 2 If $\left\langle x_{1}, x_{2} \ldots, x_{m}\right\rangle$ is any sequence of integers whose sum is 1 , then exactly one of the cyclic shifts $\left.\left\langle x_{1}, x_{2} \ldots, x_{m}\right\rangle,\left\langle x_{2} \ldots, x_{m}, x_{1}\right\rangle \ldots<x_{m}, x_{1} \ldots, x_{m-1}\right\rangle$ has all of its partial sums positive.

In the sequel we will also represent the paths of $X_{2 n+1}$, as $(2 n+1)$-vectors, obtained by encoding each rise step with 1 , each fall step by -1 , each $\alpha$-colored horizontal step with 2 , and each $\beta$-colored horizontal step with -2 . For an arbitrary path $P \in X_{2 n+1}$, let $v(P)$ denote its vectorial representation.

Since there are $\left(\begin{array}{c}2 n+1 \\ n\end{array}\right)^{2}$ paths of $X_{2 n+1}$, Lemma 2 implies that exactly $1 /(2 n+1)$ of these paths have a vectorial representation with all partial sums positive (see Fig. 8 on page 6). Let $J_{2 n+1}$ denote the set of those paths. We next establish a direct bijection between the positive cycles of length $2 n$ and paths of $J_{2 n+1}$, thus obtaining (7).

Let $P$ be a positive cycle of length $2 n$. Moreover, let $A$ be the rightmost point belonging to $P$ such that the partial sums of the vector $v(P)$ assume the lowest value, say $a,-a \leq 0$. Then $P$ can be decomposed in two sub-paths, $L$ and $R$, on the left and on the right of $A$, respectively (see Fig. 9 on page 7). It should be clear that the vector $v(R)$ has all partial sums positive. We consider the new path $P^{\prime}$ formed by transposing the paths $L$ and $R$, and adding a rise step between them. We will prove that $P^{\prime} \in J_{2 n+1}$, that is, the vector $v\left(P^{\prime}\right)$ has all partial sums positive. Let $v(L)$ and $v(R)$ be the vectors encoding $L$ and $R$ respectively. Surely, the sum of the integers of $v\left(P^{\prime}\right)$ is equal to 1 . Suppose that there is a prefix $q$ of $v\left(P^{\prime}\right)$ such that $q$ 's sum is equal to 0 . For the previous considerations $q$ must contain strictly $v(R)$, thus $q=\left(r_{1}, \ldots, r_{k}, 1, s_{1}, \ldots, s_{h}\right)$, $r_{i}, s_{i} \in\{0,1\}, v(R)=\left(r_{1}, \ldots, r_{k}\right)$, and $h \geq 1$. Therefore, since $r_{1}+\ldots+r_{k}=a>0(a=0$ if and only if $v(R)$ is empty), we must have $1+s_{1}+\ldots+s_{h}=-a$, and then $s_{1}+\ldots+s_{h}=-a-1$. Finally, the vector $s=\left(s_{1}, \ldots, s_{h}\right)$ represents a prefix $S$ of $L$, such that $v(S)=-a-1$, contradicting our initial hypothesis. Then $P^{\prime} \in J_{2 n+1}$.

The previously defined bijection can be easily inverted as follows: given a path $P^{\prime}$ in $J_{2 n+1}$, let $B$ be $P^{\prime}$ rightmost point having the lowest ordinate. The point $B$ divides $P^{\prime}$ in two sub-paths, $U$ and $V$, on the left and on the right of $B$, respectively. Let $V^{\prime}$ be the path obtained from $V$ by deleting the initial rise step, and 

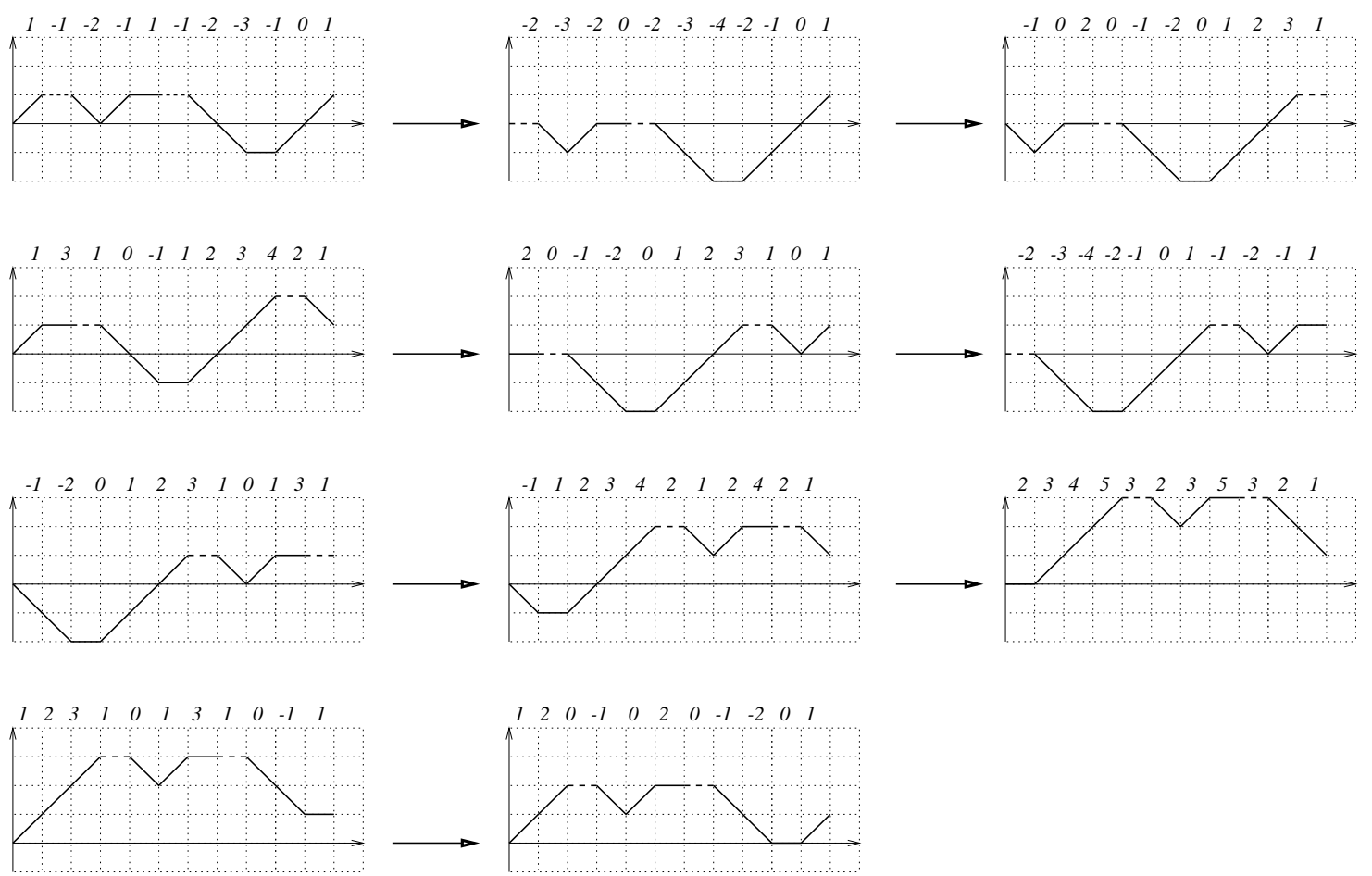

Fig. 8: The cyclic shifts of a path in $X_{2 n+1}$ and the the partial sums of the corresponding vectors.

$P$ the path obtained by transposing the paths $U$ and $V^{\prime}$; namely, $P=V^{\prime} U$. Clearly, $P$ is a positive cycle. Figure 10 on page 7 shows the bijection between the 3 positive cycles of length 2 and the 3 paths of $J_{3}$.

\section{Bijective results on directed-convex polyominoes}

A polyomino is a finite union of elementary cells of the lattice $Z \times Z$, whose interior is connected. Most of them can be defined by combining two notions: convexity and directed growth. A polyomino is said to be vertically convex when its intersection with any vertical line is convex. We can define similarly a notion of horizontal convexity. A polyomino is convex if it is both vertically and horizontally convex. A polyomino $P$ is said to be directed when every cell of $P$ can be reached from a distinguished cell, called the root, by a path which is contained in $P$ and uses only north and east unitary steps. A polyomino is directed-convex if it is both directed and convex (see Fig. 11 (a) on page 8).

A parallelogram polyomino is a polyomino whose boundary consists of two lattice paths that intersect only initially and finally. The boundary paths, which we call upper and lower path, use the positively directed unit steps, $(1,0)$ and $(0,1)$ (see Fig. 11, $(b)$ on page 8). Chang and Lin [3], and later BousquetMélou [1, p.111] used analytic methods to prove that the number of directed-convex polyominoes and the number of parallelogram polyominoes having $q$ rows and $p$ columns are equal to, respectively, 

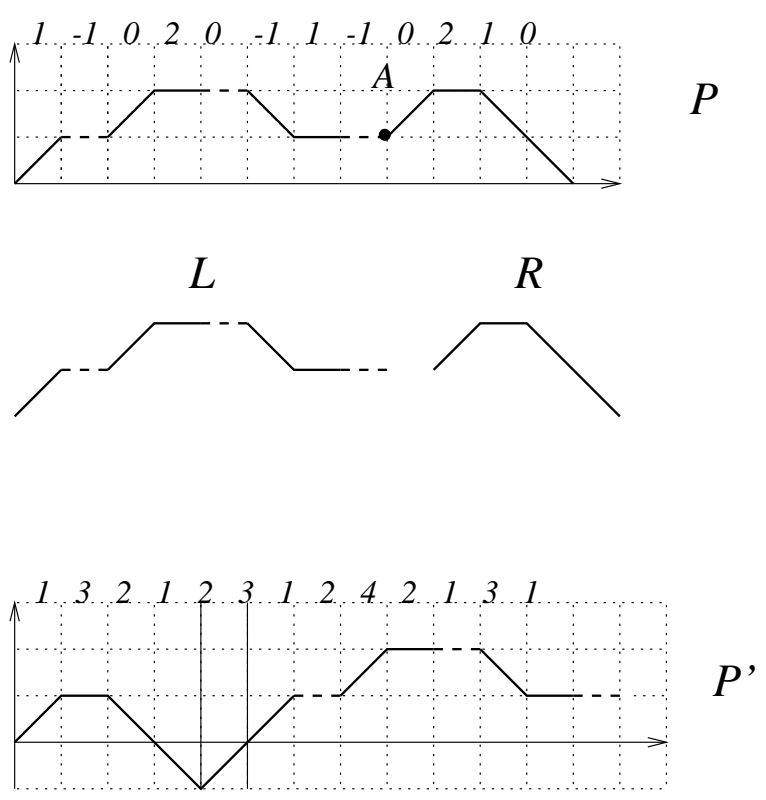

Fig. 9: A positive cycle and the corresponding path of $J_{2 n+1}$.

$$
\begin{gathered}
\left(\begin{array}{c}
p+q-2 \\
p-1
\end{array}\right)\left(\begin{array}{c}
p+q-2 \\
q-1
\end{array}\right) \\
\frac{1}{p+q-1}\left(\begin{array}{c}
p+q-1 \\
p-1
\end{array}\right)\left(\begin{array}{c}
p+q-1 \\
q-1
\end{array}\right) .
\end{gathered}
$$

(The second formula is originally due to Narayana, [9].) In particular, for polyominoes having $n+1$ rows and $n+1$ columns, these formulas reduce to

$$
\left(\begin{array}{c}
2 n \\
n
\end{array}\right)^{2}
$$

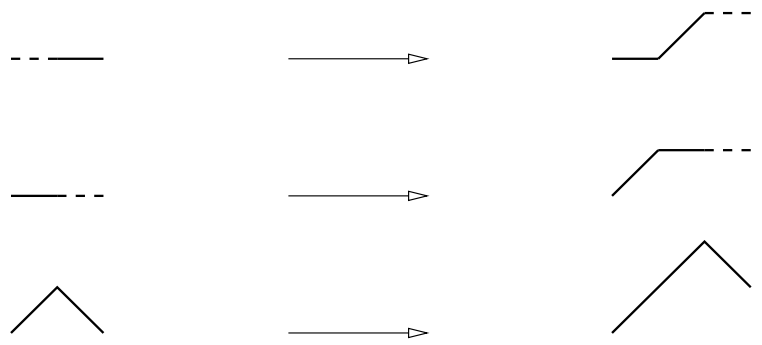

Fig. 10: The bijection between the positive cycles of length 2 and $J_{3}$. 


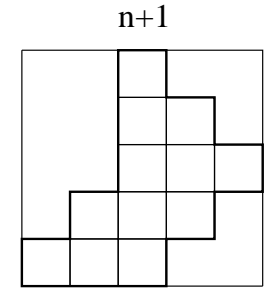

(a)

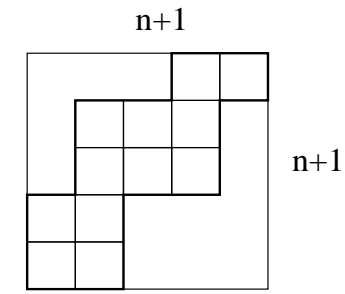

(b)

Fig. 11: (a) A directed convex polyomino; (b) a parallelogram polyomino.

$$
\frac{1}{2 n+1}\left(\begin{array}{c}
2 n+1 \\
n
\end{array}\right)^{2}
$$

respectively, that is the numbers in (1), and (2). Let us denote by $\mathcal{D} \mathcal{C}_{n}$ the class of directed-convex polyominoes having $n$ rows and $n$ columns and by $\mathcal{P} \mathcal{P}_{n}$ the class of parallelogram polyominoes having $n$ rows and $n$ columns. We will reprove (10) this time by simply establishing a bijection between the class $\mathcal{D} \mathcal{C}_{n+1}$ and $2 n$-length cycles. Similarly, we will reprove (11) by establishing a bijection from $\mathcal{P} \mathcal{P}_{n+1}$ to the class of positive cycles of length $2 n$. For this purpose, we define an auxiliary class $H_{n}$ of prefixes of positive cycles, having length $2 n$, having an equal number of $\alpha$ and $\beta$-colored horizontal steps, and having a final point with an even ordinate, say $2 h, h \geq 0$.

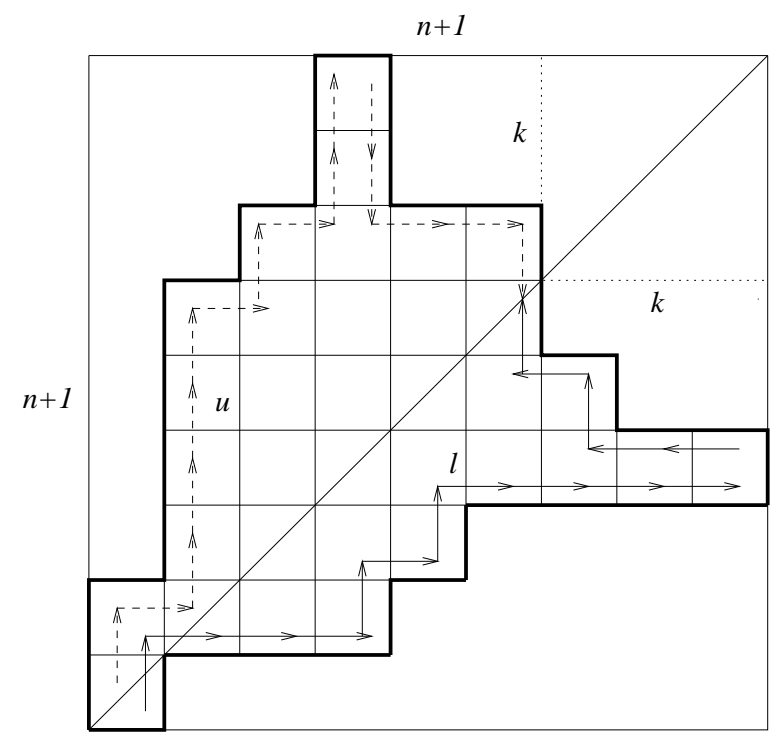

Fig. 12: A directed convex polyomino and its boundary paths. 
The bijection between $\mathcal{D C} \mathcal{C}_{n+1}$ and $H_{n}$. Consider a polyomino $P \in \mathcal{D} C_{n+1}$. Let $(k, k)$ denote the rightmost point on $P$ on the diagonal running from $(0,0)$ to $(n+1, n+1)$. We remark that each polyomino $P$ is uniquely determined by its boundary paths, the upper, say $u$, and the lower, say $l$, running from $(0,0)$ to $(k, k)$ (see Fig. 12), each path consisting in $2 n$ unit steps belonging to $\{(1,0),(0,1),(-1,0),(0,-1)\}$. Moreover, by considering the scheme of Fig. 12 on page 8, one can see that each boundary path can be represented by means of a binary array of $2 n$-elements where 0 represents the steps $(1,0)$ and $(-1,0)$ and 1 the steps $(0,1)$ and $(0,-1)$. It follows that the polyomino $P$ can be represented by a $2 \times n$ binary matrix, where the first row corresponds to the upper boundary path and the second corresponds to the lower one. For example, the polyomino of Fig. 12 on page 8 can be represented by the matrix:

$$
\left(\begin{array}{llllllllllllllll}
1 & 0 & 1 & 1 & 1 & 1 & 0 & 1 & 0 & 1 & 1 & 1 & 1 & 0 & 0 & 1 \\
1 & 0 & 0 & 0 & 1 & 0 & 1 & 0 & 0 & 0 & 0 & 0 & 0 & 1 & 0 & 1
\end{array}\right) .
$$

We wish to point out two properties of the upper and lower paths, $u$ and $l$ :

1. for every prefix $s$ of $u$ and every prefix $v$ of $l$, having the same length, we have $|s|_{1} \geq|v|_{1}$, with $|j|_{1}$ defined as the number of occurrences of 1 in $j$;

2. $|u|_{1}-|l|_{1}=2 k$.

Besides, the matrix can be viewed as an array of $n$ vectors $2 \times 1$. Then, it is possible to represent it as path $P^{\prime}$ belonging to $H_{n}$, and whose final point ordinate is equal to $2 k$, by means of the coding defined for the cycles in the proof of Lemma 1 . For example, Figure 13 represents the $H_{n}$ path corresponding to the polyomino in Fig. 12 on page 8.

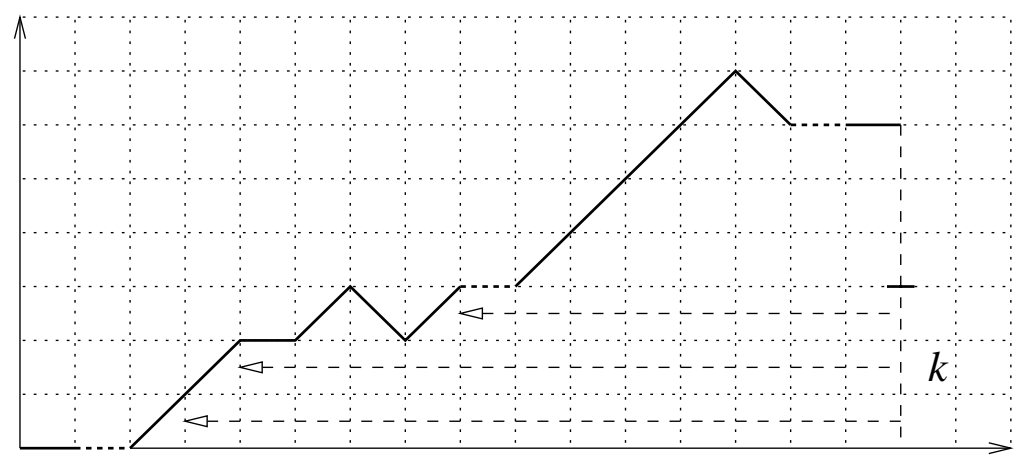

Fig. 13: The $H_{n}$ path corresponding to the polyomino in Fig. 12 on page 8.

It should be clear that this mapping from directed-convex polyominoes having $n+1$ rows and $n+1$ columns to the paths of $H_{n}$ can be easily inverted. In the special case that $P$ is a parallelogram polyomino we have $|u|_{1}-|l|_{1}=0$; that is, we have the desired correspondence between parallelogram polyominoes and positive cycles (see Fig. 14 on page 10). We wish to point out that the last bijection is a special case of a classical bijection between parallelogram polyominoes of perimeter $2 n+4$ and 2-colored Motzkin paths of length $2 n$ [4]. 
$n$
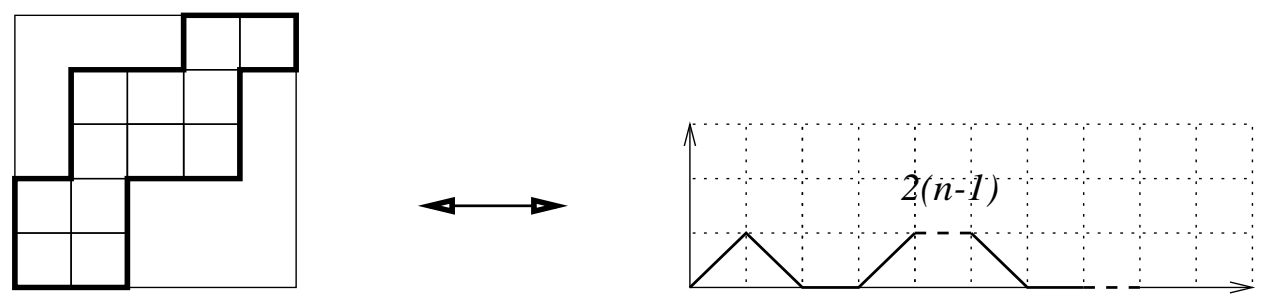

Fig. 14: A particular case of the bijection is the restriction to parallelogram polyominoes and positive cycles.

The bijection between $H_{n}$ and $2 n$-length cycles. Let $P^{\prime}$ be a path in $H_{n}$ and let $2 k, k \geq 0$, be its final point ordinate. If $k=0$, then $P^{\prime}$ is a positive cycle. Otherwise, for every $i=0, \ldots, k-1$ we consider the vertical side of unitary length $(2 n, i),(2 n, i+1)$. We then draw a horizontal ray to the left from the center of this side. There are $k$ such rays. Each ray hits for the first time a rise step in $P^{\prime}$. We modify $P^{\prime}$ by

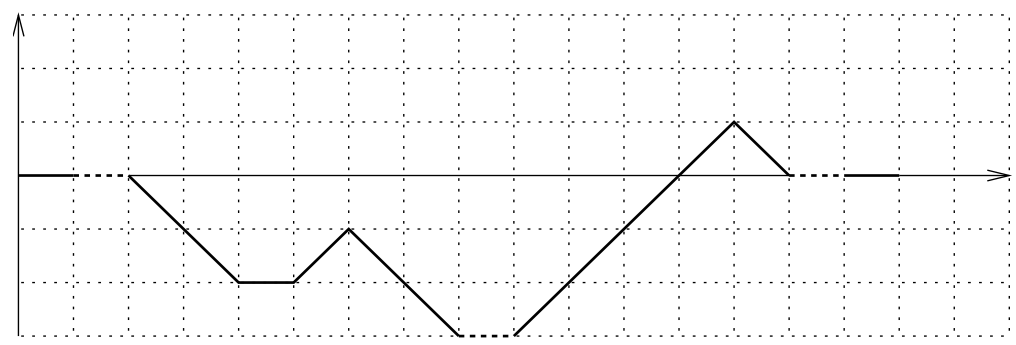

Fig. 15: The cycle corresponding to the $H_{n}$ path in Figure 14.

changing the steps that are hit to fall steps. In this modified path the number of rise step is trivially equal to the number of fall steps, thus we have obtained the desired cycle (see Fig. 15 on page 10).

This mapping is inverted as follows (see Fig. 16 on page 11). Let $Q$ be a $2 n$-length cycle and let $-h, h>0$ be the ordinate of the lowest point of $Q$. From each of the points $\left(0,-\frac{1}{2}\right),\left(0,-1-\frac{1}{2}\right), \ldots$, $\left(0,-h+1-\frac{1}{2}\right)$, we draw a ray to the right until it hits $Q$, necessarily at a fall step. Let $Q^{\prime}$ be the path obtained from $Q$ in which each hit step is changed to a fall step. The path $Q^{\prime} \in H_{n}$, and its final point ordinate is equal to $2 h$.

\section{Conclusions}

In this paper we essentially described:

1. the correspondence among the class of lattice paths using north, south, east, and west steps, beginning and ending at $(0,0)$; the class of 2-colored Motzkin paths having the same number of $\alpha$ and $\beta$-colored steps; and the class of directed-convex polyominoes having the same number of rows and columns. That correspondence leads to a combinatorial interpretation of the numbers in (1);

2. the correspondence among the class of lattice paths using north, south, east, and west steps, beginning and ending at $(0,0)$ remaining weakly above the $x$-axis; the class of 2-colored Motzkin paths 

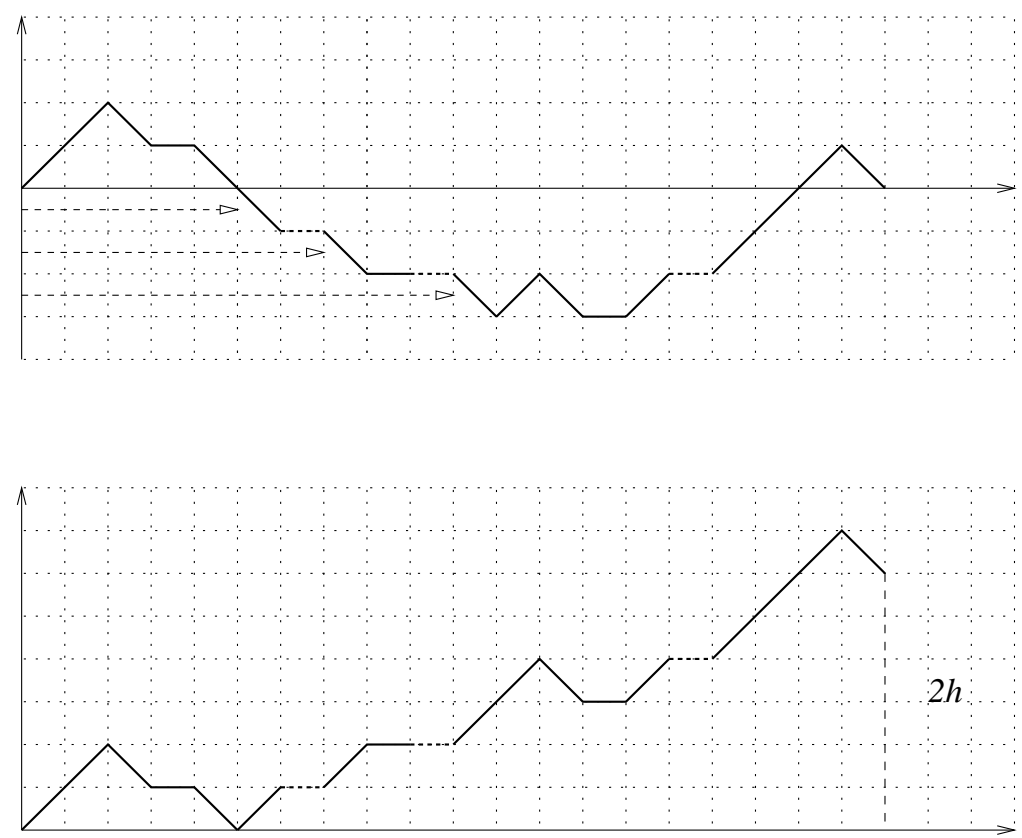

Fig. 16: From a $2 n$-length cycle to a $H_{n}$ path.

having the same number of $\alpha$ and $\beta$-colored steps, remaining weakly above the $x$-axis; and the class of parallelogram polyominoes having the same number of rows and columns. That correspondence leads to a combinatorial interpretation of the numbers in (3).

We observe that it is possible to generalize the correspondences 1 . and 2. to

1. the class of lattice paths using north, south, east, and west steps, beginning at $(0,0)$ and ending in $(p-q, 0), p, q \in N$, made by $p+q-2$ steps, (resp. the paths remaining weakly above the $x$-axis);

2. the class of 2-colored Motzkin paths of length $p+q-2$, such that the difference between the number of $\alpha$ and $\beta$-colored steps is equal to $p-q$ (resp. the paths remaining weakly above the $x$-axis);

3. the class of directed-convex polyominoes having $p$ rows and $q$ columns (resp. the class of parallelogram polyominoes having $p$ rows and $q$ columns)

thus giving combinatorial proofs of the formulas (10) and (11).

\section{Acknowledgements}

Authors wish to thank Robert A. Sulanke for many helpful suggestions and comments. 


\section{References}

[1] M. Bousquet-Mélou, $q$-Énumération de polyominos convexes, Publications du L.A.C.I.M. (1991).

[2] W. Breckenridge, H. Gastineau-Hills, A. Nelson, P. Bos, G. Calvert, and K. Wehrhahn, Lattice paths and Catalan numbers, Bull. Inst. Comb. and its App., 1 (1991) 41-55.

[3] S. J. Chang, and K. Y. Lin, Rigorous results for the number of convex polygons on the square and honeycomb lattices, J. Phys. A: Math. Gen., 21 (1988) 2635-2642.

[4] M. Delest, and X. Viennot, Algebraic languages and polyominoes enumeration, Theor. Comp. Sci., 34 (1984) 169-206.

[5] N. Dershowitz and S. Zaks, The cycle lemma and some applications, Europ. J. Comb., 11 (1990) $35-40$.

[6] N. Dvoretzky and T. Motzkin, A problem of arrangements, Duke Math. J., 14 (1947) 305-313.

[7] R. L. Graham, D. E. Knuth, and O. Patashnik, Concrete mathematics, Addison-Wesley Publishing Company, New York (1989).

[8] R. K. Guy, C. Krattenthaler, and B. Sagan, Lattice paths, reflections and dimension-changing bijections, Ars Combinatorica, 34 (1992) 3-15.

[9] T. V. Narayana, Sur les treillis formés par les partitions d'un entier; leurs applications à la théorie des probabilités, Comp. Rend. Acad. Sci. Paris, 240 (1955) 1188-9.

[10] G. M. Raney, Functional composition patterns and power series reversion, Trans. Am. Math. Soc., 94 (1960) 441-451 .

[11] N. J. A. Sloane, and S. Plouffe, The Encyclopedia of Integer Sequences, Academic Press, New York (1995).

[12] R. P. Stanley, Enumerative Combinatorics, Vol.2, Cambridge University Press, Cambridge (1999).

[13] R. A. Sulanke, A note on counting lattice walks restricted to the half plane, (preprint). 